\title{
Methods of Resistance Estimation in Permanent Magnet Synchronous Motors for Real-Time Thermal Management
}

\author{
Simon D. Wilson, Paul Stewart Senior Member, IEEE, and Benjamin P. Taylor
}

\begin{abstract}
Real-time thermal management of electrical machines relies on sufficiently accurate indicators of internal temperature. One indicator of temperature in a permanent-magnet synchronous motor (PMSM) is the stator winding resistance. Detection of PMSM winding resistance in the literature has been made on machines with relatively high resistances, where the resistive voltage vector is significant under load. This paper describes two techniques which can be applied to detect the winding resistance, through 'Fixed Angle' and 'Fixed Magnitude' current injection. Two further methods are described which discriminate injected current and voltages from motoring currents and voltages: 'Unipolar' and 'Bipolar' separation. These enable the resistance to be determined, and hence the winding temperature in permanent-magnet machines. These methods can be applied under load, and in a manner that does not disturb motor torque or speed. The method distinguishes between changes in the electro-motive force (EMF) constant and the resistive voltage. This paper introduces the techniques, whilst a companion paper covers the application of one of the methods to a PMSM drive system.
\end{abstract}

Index Terms-Resistance measurement, temperature measurement, temperature control, parameter estimation.

\section{INTRODUCTION}

B RUSHLESS permanent-magnet machines have emerged as the preferred electrical machine technology for many applications due to their high specific power and efficiencies. In common with other types of electrical machine, their output power is usually limited by the maximum operating temperature of key machine components [1]. Of particular significance are the lifetime of coil insulation which reduces markedly beyond the manufacturers specified temperature rating [2], and the diminishing magnetic properties of the permanent magnets. To ensure that the maximum temperature limit is not exceeded during service, it is common practice to design a machine on the basis of a worst-case estimate of the operating conditions, which amongst over things, should account for the highest anticipated ambient temperature, worst-case load conditions

At the time of this research the authors were with the Electrical Machines and Drives Research Group, The University of Sheffield, Mappin Street, S1 3JD, U.K. S.D. Wilson is now with EA Technology, Capenhurst Technology Park, Capenhurst, CH1 6ES. Paul Stewart is now with the Univeristy of Lincoln (e-mail: simon.wilson@eatechnology.com; pstewart@lincoln.ac.uk; b.p.taylor@shef.ac.uk).

S.D. Wilson was supported by a grant from the 'Engineering and Physical Sciences Research Council' (EPSRC) and a 'Co-operative Awards in Science and Engineering' (CASE) award provided by Rolls-Royce PLC. Technical support, and the hardware used in this research was provided by the EPSRC Project 'Zero-Constraint Free Piston Energy Converter', Grant Number: GR/S97507/01. and most onerous duty cycle. An inevitable consequence of this approach to machine rating is that it will operate well below its potential for much of its service life, particularly in applications with intermittent or unpredictable duty-cycles. In such applications, a machine could achieve a transient power rating that is considerably greater than its continuous rating if the thermal capacity of the machine is fully harnessed (although it is essential to ensure that the magnet has been adequately dimensioned to avoid irreversible demagnetisation). This said, it should also be recognized that under extreme short-term overload conditions, increases in output power are accrued with diminishing return as a consequence of the onset of magnetic saturation in the stator core.

The key to reliably and fully exploiting the thermal capacity of a machine in applications with variable and often unpredictable duty cycles, is monitoring key temperatures within the machine. Temperature monitoring within a machine could be achieved using an array of thermal sensors embedded throughout the machine. One drawback of this approach is that the relationship between the sensor and component temperatures is reliant on the interface properties, particularly in terms of any time-lags introduced. Moreover, an array of embedded sensors increases the number of connections required between the motor and its controller, with a detrimental impact on system reliability [3]. A so-called 'sensorless' technique is therefore attractive, whereby estimates of stator coil temperatures can be obtained by tracking changes in the coil resistance, which in turn can be derived from terminal voltage and current measurements.

Although terminal resistance measurements only provide a means of determining average coil temperatures, these inferred temperatures could in principle be used as input to a machine specific thermal model to provide more localized temperature information, e.g. track any hotspot in the end-windings. As well as providing an effective means of protection against over-temperature, the accurate tracking of winding temperature provides a valuable source of information for health monitoring and prognostics and offer the potential for real-time thermal management of a machine, e.g. real-time variable derating to match peak transient capability in unexpected service conditions.

Although the relatively high sensitivity of coil resistance to temperature rise would appear to provide a convenient means of estimating stator coil temperature, in practice tracking resistance changes in PM machines is extremely challenging, particularly in high efficiency, high power machines. In such 
machines, the contribution from the stator resistive voltage drop to the net terminal voltage under normal operating conditions is often very small, typically being of the order of less than a few percent. By way of illustration the target motor for this research similarly has a resistive voltage drop at rated current of just 1.1 percent of the terminal voltage, with the parameters of Table I. In order to provide a temperature resolution as coarse as $10^{\circ} \mathrm{C}$ in this machine, a resolution which might be regarded as the upper limit for tracking temperatures to avoid transient overheating, would require a means of reliably extracting the true resistive voltage component from terminal measurements to a resolution of 0.04 percent. These difficulties are compounded in a practical drive system by the presence of several disturbances in the voltage and current waveforms due to switching ripple, the action of closed-loop controllers which continually adjust the current to meet some specified commanded motion, the presence of varying degrees of magnetic saturation in the stator and/or rotor core (this being particularly problematic under severe short-term overload) and rotor saliency.

As a consequence, resistance estimation methods that are solely reliant on terminal measurements under normal operating conditions cannot usually provide the precision required for high power PM machines. The method described in this paper is based on intermittent current injection to temporarily boost the resistive voltage contribution, thus alleviating to some degree the difficulties of ensuring reliable measurement. Since the thermal time constant of a large PM machine (and hence the frequency with which estimates of resistance need to be obtained) is many orders of magnitude longer than the duration of injected pulses (which are typically tens of milliseconds), then the additional heating effect within the machine is negligible. However, it is necessary in many applications to ensure that the transient disturbance to the motor normal operation during the current injection is minimized.

\section{Stator-Winding RESistance Estimation}

The stator winding of a typical machine consists of several insulated copper coils, whose resistance varies as a function of coil temperature as:

$$
R_{s}=R_{s 0}+\alpha R_{s 0}\left(T_{s}-T_{0}\right)
$$

where $R_{s 0}$ is the winding resistance at $T_{0}{ }^{\circ} \mathrm{C}, R_{s}$ is the winding resistance at $T_{s}{ }^{\circ} \mathrm{C}$, and $\alpha$ is the temperature coefficient of copper $\left(3.93 \times 10^{-3}\right.$ per $\left.{ }^{\circ} \mathrm{C}\right)$.

For $R_{s 0}=0.133 \Omega$ at $25^{\circ} \mathrm{C}, R_{s}=0.183 \Omega$ at $120^{\circ} \mathrm{C}$. Thus there is significant change in resistance over an operating temperature range, even if the resistance itself is small. Detection of this change in resistance provides a means of determining the temperature of the stator coils. For a $10^{\circ} \mathrm{C}$ resolution in the operating temperature range the resistance must be estimated to an accuracy of approximately $5 \mathrm{~m} \Omega$. Achieved accuracies of $10 \mathrm{~m} \Omega$ result in a resolution of $20^{\circ} \mathrm{C}$, etc. In conjunction with thermal models, temperatures at other locations in a machine can be estimated. Temperature estimates are desired for motor protection functions and applications which require motors to be operated at the limit of their temperature envelope.
Considerable research has been published on the estimation of stator resistance in induction machines for the improvement of sensorless speed and torque control [4]-[9] and thermal monitoring [10]. On-line stator resistance estimation has also been applied to PMSMs to improve sensorless control [11], but thermal tracking has not been demonstrated.

Sensorless thermal monitoring has been demonstrated [12], protecting a $200 \mathrm{~W}$ PMSM from coolant failure. This method was based on least-squares estimation of the resistance. Despite the low power rating, and hence the more significant contribution of the resistive voltage drop, even in this case reliable estimates were only achieved over a limited speed range, beyond which the contribution of the induced EMF degraded the accuracy of the method employed.

One method of increasing the magnitude of the resistive voltage drop relative to the remaining contributors to terminal voltage, so as to aid the accuracy of resistance estimates, is to periodically inject sensing current pulses of a prescribed form into windings. Since most large, high-power machines have relatively long thermal time constants (significant temperature excursions occurring over time scales of the order of tens of seconds) it is usually only necessary to inject short sensing pulses on a very low duty cycle, e.g. tens of millisecond duration pulses every few tens of seconds.

Hence, even with sense pulses that are significant in magnitude when compared to the rated current, the additional losses generated in the machine will be marginal. However such an approach may have an impact on the converter rating, as the relatively low thermal mass of the switching elements may dictate that a substantial increase in short-term rating is necessary.

The injection of current pulses to aid resistive voltage measurements can be achieved by superimposing a bias voltage on the stator supply voltage [13], or by imposing a rotating voltage on the neutral point of a star-connected machine with a capacitor bank [14].

The first method induces a fixed current vector in the machine, which causes the motor torque output to include an oscillatory component. As the amplitude of the component increases, the accuracy of the estimate also increases. In servo systems this speed disturbance may prevent a system from accurately tracking a position or velocity profile, and makes the application of this method subject to mechanical system constraints. The lower the inertia of the system, the greater the possible speed disturbance. If the motor is connected to the load through a system with backlash, such as gears, then the system may wear faster than expected.

The second method requires the addition of a capacitor bank and access to the motor star point, which requires modification to the motor, and may not be easily accessible. Both injection methods dissipate power within the machine and any additional devices required to induce the sense currents. In [13] the average power dissipation of the DC injection method is shown to be negligible. The method presented in this article, overcomes these two deficiencies by using the motor control system to inject the sense current along the direct-axis of the machine. 


\section{PMSM DQ-AXIS MODEL}

The concept of resolving machine armature quantities into two rotating components, one aligned with the field axis, the direct-axis component $(d)$, and one in quadrature with the field axis, $(q)$ is a well-established and widely used means of analyzing electrical machines. These components, which are stationary with respect to the rotor, are referred to as being in the rotor-stationary frame of reference (qdr). When transferred to the stator-stationary frame of reference, they are referred to as being in the stator-stationary frame of reference (qds).

The transformation itself can be represented in terms of the electrical angle between the rotor direct-axis and the stator phase-a axis [15]. Letting $S$ represent the quantity to be transformed (current, voltage or flux), the transformation can be written in matrix form using Park's transformation as

$$
\left[\begin{array}{c}
S_{d} \\
S_{q} \\
S_{0}
\end{array}\right]=
$$

$\frac{2}{3}\left[\begin{array}{ccc}\cos (\theta) & \cos (\theta-2 \pi / 3) & \cos (\theta+2 \pi / 3) \\ -\sin (\theta) & -\sin (\theta-2 \pi / 3) & -\sin (\theta+2 \pi / 3) \\ 1 / 2 & 1 / 2 & 1 / 2\end{array}\right]\left[\begin{array}{c}S_{a} \\ S_{b} \\ S_{c}\end{array}\right]$

where $S_{d}, S_{q}$ and $S_{0}$ denote the d-axis, q-axis and zerosequence components of the transformed phase quantities $S_{a}$, $S_{b}$ and $S_{c}$.

An equivalent inverse transform exists which transfers the dq0 quantities into stator three-phase quantities. Under balanced conditions there are no zero-sequence components. When the PMSM three-phase motor equations are also transformed into the rotor reference frame the motor variables may be represented by the following matrix

$$
\begin{aligned}
{\left[\begin{array}{c}
v_{d r} \\
v_{q r}
\end{array}\right] } & =\left[\begin{array}{cc}
R_{s} & -\omega L_{q} \\
\omega L_{d} & R_{s}
\end{array}\right]\left[\begin{array}{c}
i_{d r} \\
i_{q r}
\end{array}\right] \\
& +\left[\begin{array}{cc}
L_{d} & 0 \\
0 & L_{q}
\end{array}\right] \frac{d}{d t}\left[\begin{array}{c}
i_{d r} \\
i_{q r}
\end{array}\right]+\left[\begin{array}{c}
0 \\
K_{e} \omega_{m}
\end{array}\right]
\end{aligned}
$$

where $v_{d r}$ and $v_{q r}$ are the direct and quadrature axes voltages in the rotor reference frame, $i_{d r}$ and $i_{q r}$ are the direct and quadrature axes currents in the rotor reference frame, $R_{s}$ is the stator resistance, $L_{d}$ and $L_{q}$ are the direct and quadrature axis inductances, $K_{e}$ is the back-EMF constant, $\omega$ is the electrical frequency and $\omega_{m}$ is the rotor angular velocity in radians per second.

\section{DC INJECTION}

DC Injection is the superposition of a DC offset in one or more of the motor phases. The DC offset is used since it facilitates the separation by filters of the injection voltage from the motor's normal operating voltages under current control.

In principle, the sensing current can be injected into any combination of phases in a variety of relative proportions. By way of example, Fig. 1(a) shows a case in which the current is injected into phase 'A' returning entirely through phase ' $\mathrm{B}$ ' while in Fig. 1(b) the current is again injected into phase ' $A$ ' but returns in equal proportions through phases

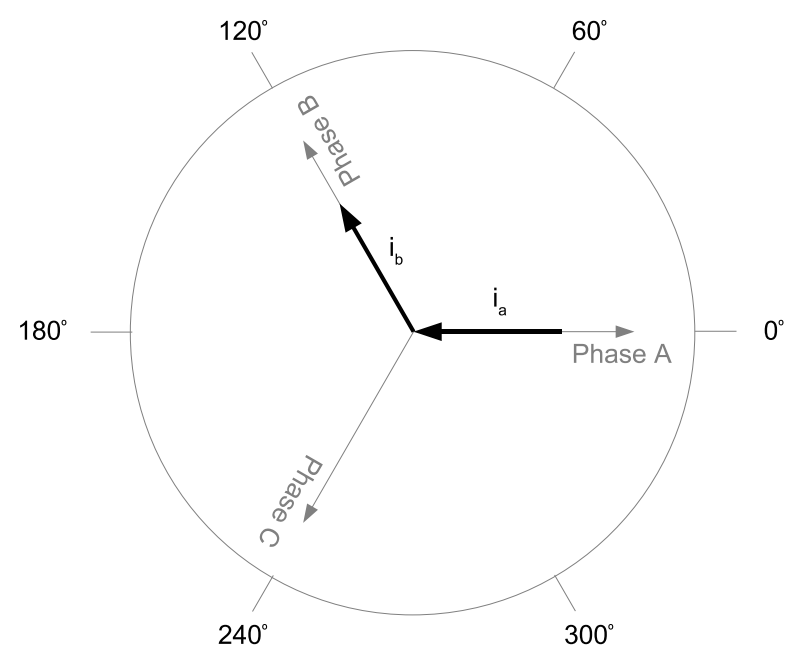

(a) Two-phase

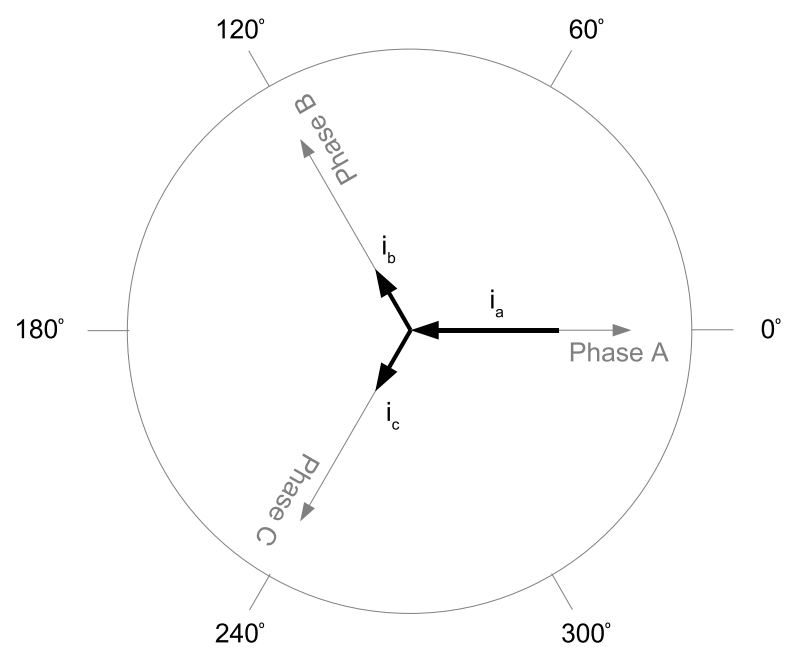

(b) Three-phase

Fig. 1. Injection Current Paths

' $\mathrm{B}$ ' and ' $\mathrm{C}$ '. Both these paths can be represented by a current vector, Fig. 2 with dq components in the qds reference frame

$$
\begin{aligned}
& i_{d s}=F \cos \left(\theta_{F}\right) \\
& i_{q s}=F \sin \left(\theta_{F}\right)
\end{aligned}
$$

where $i_{d s}$ and $i_{q s}$ are the direct and quadrature axes currents in the stator reference frame, $F$ is the magnitude of the injected current vector and $\theta_{F}$ is the angle of the injected current vector.

The injected current causes a magneto-motive force to be present in the airgap of the machine. An additional rotating current controlled vector will be present when the motor comprises part of a drive system and is producing torque, Fig. 3. The drive system is a closed-loop current control system which minimizes the error between the axes current feedback and current demands $i_{q r}^{*}, i_{d r}^{*}$. The feedback axes currents are generated by measuring the three phase currents and transforming them into dq-frame (Eq. 3). The outputs of the current controller are the calculated demand voltages $v_{q r}^{*}, v_{d r}^{*}$, which are transformed into the phase voltage demands via the 


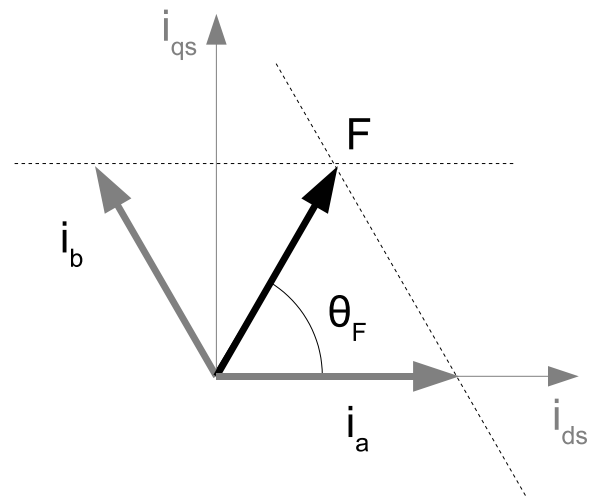

Fig. 2. General Current Vector

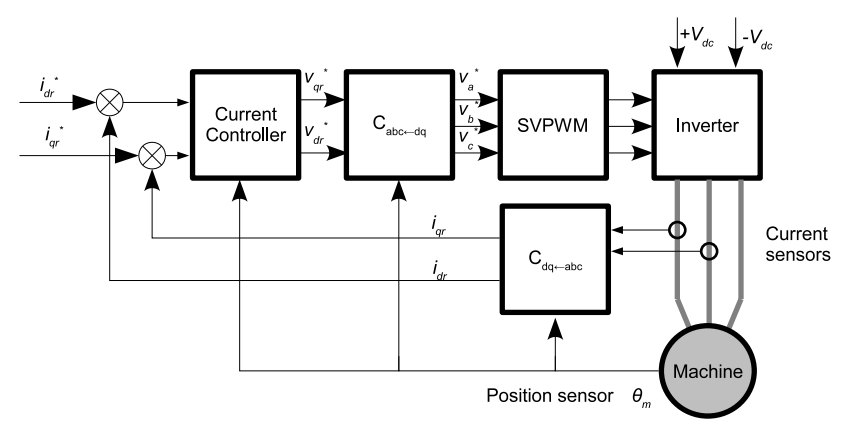

Fig. 3. Schematic of Current-Controlling Drive System

'Space-Vector Pulse-Width Modulation' (SVPWM) algorithm and the inverse transform of Eq. 3. The three-phase inverter is supplied with bus voltages $+V_{d c},-V_{d c}$ and applies the relevant switching voltages to the motor.

As the injection currents must be superimposed with the rotating current vector, the application of current injection must be managed in order to prevent the total current exceeding the motor limit. When referred to the dq or 'rotor stationary frame'(qdr), the injection current vector is represented as rotating

$$
\begin{aligned}
& i_{d r}=F \cos \left(\theta_{F}-\theta\right) \\
& i_{q r}=F \sin \left(\theta_{F}-\theta\right)
\end{aligned}
$$

where $\theta$ is the rotor electrical angle. The following equations of d- and q-axis voltage are obtained by substitution of (Eq. 6 \& 7) in (Eq. 3)

$$
\begin{aligned}
v_{d r}= & F R_{s} \cos \left(\theta_{F}-\theta\right) \\
& -\omega F\left(L_{d}+L_{q}\right) \sin \left(\theta_{F}-\theta\right) \\
v_{q r}= & F R_{s} \sin \left(\theta_{F}-\theta\right) \\
& +\omega F\left(L_{d}+L_{q}\right) \cos \left(\theta_{F}-\theta\right)
\end{aligned}
$$

The resistive and inductive parts generate voltage vectors which are orthogonally related. The resistance and inductance can therefore be estimated separately by resolving along angles $\theta-\theta_{F}$ and $\theta-\theta_{F}+90^{\circ}$. The loci of the vectors is presented in Fig. 4, where $\left|V_{R}\right|=F R_{s},\left|V_{L}\right|=\omega F\left(L_{d}+L_{q}\right)$.

The injection currents and detected voltages comprise the DC injection scheme, similar to that implemented by Lee

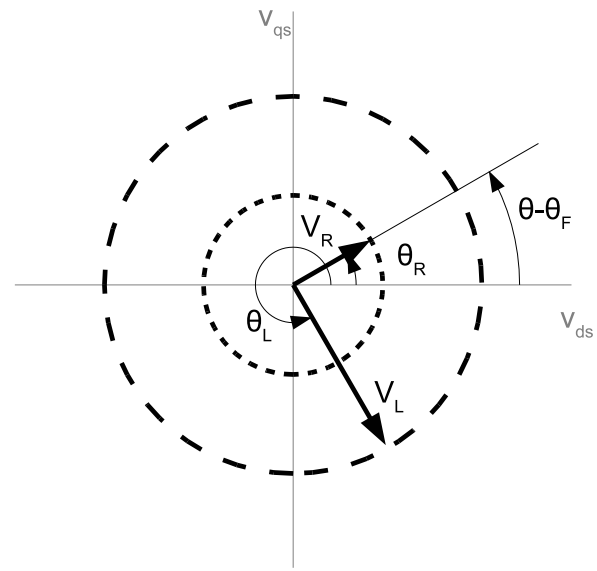

Fig. 4. DC Injection Resistance and Inductance Voltage Loci

\& Habetler [13] but represented in the dq-frame. The injection voltage generated is stationary with respect to the stator.

For stator current magnitudes below those at which there is appreciable magnetic saturation of the stator and/or rotor core, the torque produced by a PMSM can be represented in dq-axis variables by

$$
T_{e m}=K_{t} i_{q r}
$$

where $T_{e m}$ is the torque and $K_{t}$ is the torque-constant of the PMSM

The DC-injection method must therefore produce a torque ripple, as both d- and q-axis currents are generated (Eq. 6 $\& 7$ ). For accurate resistance estimation, the magnitude of the injection current must be large. This causes large torque ripples, so the engineer must constrain the current injection magnitude $F$ (and therefore accuracy) to the limit of the connected mechanical system.

This torque ripple can cause significant speed deviation during the application of the DC offset. The consequence of the distortion on connected mechanical systems could be severe, especially at low loads since the output torque polarity could reverse. The speed deviation caused cannot be corrected by the motor controller, since it would necessitate cancellation of the q-axis current and consequent deformation of the injection current.

Thus the scope for application of the DC Injection method is limited to systems with large inertial loads and stiffly connected mechanisms. In order to prevent the injected sense current unduly disturbing the electromagnetic torque, it is necessary to adopt a current injection method which takes due cognizance of the effect on the instantaneous output torque.

\section{Direct-Axis Injection Methods}

\section{A. Fixed-Angle Current Injection}

This method represents a development of the DC-injection method as presented in Section I. The injection currents are modified in order to reduce or eliminate the unwanted torque pulsation. Reduction of the q-axis injection current by the regulation factor $\beta$ causes a corresponding reduction in torque 
pulsation. The injection currents and torque production can be formulated

$$
\begin{aligned}
i_{d r} & =F \cos \left(\theta_{F}-\theta\right) \\
i_{q r} & =\beta F \sin \left(\theta_{F}-\theta\right) \\
T_{e m} & =\beta F K_{t} \sin \left(\theta_{F}-\theta\right)
\end{aligned}
$$

where $T_{e m}$ denotes the pulsating torque produced by injection.

For $\beta=0$ there is no torque production and the axes voltages due to the injection current (Eq. 11) are described

$$
\begin{aligned}
& v_{d r}=F R_{s} \cos \left(\theta_{F}-\theta\right)-\omega F L_{d} \sin \left(\theta_{F}-\theta\right) \\
& v_{q r}=\omega F L_{d} \cos \left(\theta_{F}-\theta\right)
\end{aligned}
$$

Because the current vector achieves a fixed angle with respect to the rotor in the stator-stationary frame, it is referred to as 'Fixed-Angle Current Injection'. The general disadvantage of the fixed angle is that the magnitude of the sense current must vary. The resistance can best be estimated when the voltage $F R_{s}$ is a maximum, so estimating the resistance at a rotor angle other than $\theta=\theta_{F}$ is less than optimal.

\section{B. Fixed-Magnitude Current Injection}

The alternative formulation of current injection is to maintain a constant magnitude of injection current but to allow the phase to vary such that the criterion of zero torque production is retained. Thus a constant magnitude of resistive voltage with respect to angle is achieved. This is a desirable feature, allowing estimated data to be combined to form a single preferred value representing resistance. The currents are formulated in the qds frame

$$
\begin{aligned}
& i_{d s}=F \cos (\theta) \\
& i_{q s}=F \sin (\theta)
\end{aligned}
$$

Hence the magnitude is constant, and the angle varies with rotor position. When transferred to qdr frame

$$
\begin{aligned}
& i_{d r}=F \\
& i_{q r}=0
\end{aligned}
$$

If the method is restricted to purely negative $F$, then this method becomes analogous to the 'Flux-Weakening' method of extending the controllable speed range of a motor under the constraint of some finite applicable voltage magnitude [16]. The estimation voltages are obtained by substitution of the $q d r$ frame injection currents (Eq. 18 \& 19) into the PMSM voltage equation (Eq. 3)

$$
\begin{aligned}
& v_{d r}=F R_{s} \\
& v_{q r}=\omega F L_{d}
\end{aligned}
$$

The resistive and inductive voltages produced by injection are naturally separated onto the $d \& q$-axes respectively. Notably the resistive voltage is orthogonally separated from the inductive voltage and the EMF. This is advantageous considering that the resistive voltage is generally the smallest of the three quantities in magnitude.

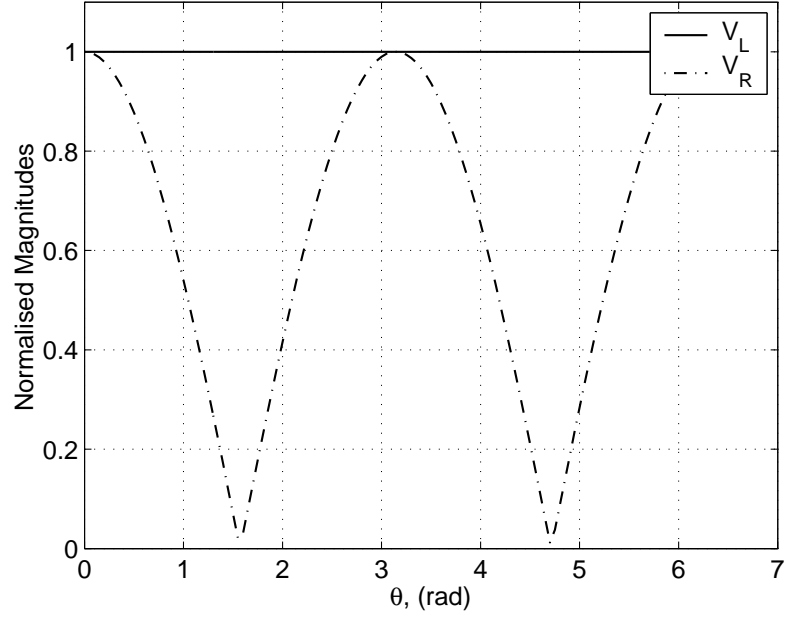

Fig. 5. Normalized Magnitudes of Injection Voltages for $\theta_{F}=0^{\circ}$

\section{Signal Voltage Analysis}

\section{A. Fixed-Angle}

To realize the most accurate estimator performance per ampere of injected current, the largest magnitude and most significant contribution from the resistive voltage term to the terminal voltage is required.

The resistive voltage term in (Eq. 14) is maximum at $\theta=\theta_{F}$, coinciding with the maximum of the d-axis injection current (Eq. 11). The inductive voltage magnitude is constant, and the two voltages are orthogonally related. As rotor position moves away from $\theta=\theta_{F}$, the angle between the two voltages becomes non-orthogonal. Therefore to estimate the resistance most accurately requires the rotor position to be $\theta=\theta_{F}$. Both the resistance and inductance can be estimated at this point.

While the machine is rotating there will be a short time interval during which the greatest resistive signal voltage is available. This is the best rotor position to estimate the resistance, but exists momentarily. To account for the existence of noise and other possible error voltages in the measurement it is prudent to increase the arc of rotor angle (and therefore the number of measurements) for which the resistance can be estimated. With small arcs of rotor angle the difference in magnitude of the injection current is small. For example with a $30^{\circ}$ arc centered on the optimal angle, the difference in current magnitude is only 3.4 percent. The signal voltages are resolved when not orthogonal by the equations

$$
\left[\begin{array}{l}
V_{R} \\
V_{L}
\end{array}\right]=\left[\begin{array}{c}
\frac{-V_{q d s} \sin \left(\theta_{L}-\theta_{q d s}\right)}{\sin \left(\theta_{R}-\theta_{L}\right)} \\
\frac{V_{q d s} \sin \left(\theta_{R}-\theta_{q d s}\right)}{\sin \left(\theta_{R}-\theta_{L}\right)}
\end{array}\right]
$$

where $V_{q d s}, \theta_{q d s}$ are the magnitude and angle of the sum of the resistive and inductive voltages in the $q d s$ frame, and $\theta_{R}, \theta_{L}$ are the angles of the resistive and inductive voltages respectively.

The denominator of the equation becomes zero when $\theta_{R}=$ $\theta_{L}$, with rotor position $\theta=\theta_{F}+\pi / 2$. This condition will not be satisfied for useful resistance estimates since the resistive signal voltage is zero at this angle. $\theta_{R}$ and $\theta_{L}$ can be calculated from (Eq. $14 \& 15$ ), and depend on rotor position but not on the magnitudes of $R_{s}$ or the inductances. 
The injection current should be concentrated in the region of greatest accuracy to prevent excessive losses. The required current is an arc, which can be produced by using a window function multiplied by the injection current (Eq. 11).

\section{B. Fixed-Magnitude}

Since the injection current is not defined by rotor angle in the qdr frame, a constant-magnitude resistive voltage is generated with respect to angle. As such there is no rotor position which offers the greatest resistive voltage, and no requirement to limit the application of current around this point. The use of a window function is prudent in limiting the voltage required to change from pre-injection motoring currents to injection currents and vice-versa.

\section{A Window Function For InJECTING CURRENT PULSES}

If the current injection pulses are crudely applied, for example by switching them on and off according to rotor position, the step currents produced may excite electrical circuit resonances, encouraging voltage oscillations to form. Excitation of the resonances can be reduced by controlling the transition from one current level to another. This control takes the form of a window function, which has a profile defined to reduce the magnitude of the voltage harmonics required to move from one current level to another [17].

By choosing a defined window function, the voltage required to make the transition can be calculated. This is useful in calculating the greatest injection current that may be applied at a given speed, or in rating the inverter.

The window function profile specifies a compromise between the voltage requirement and the length of time spent in the window period. A soft-start function such as the 'Blackman' window function can be used to minimize oscillations in the transition from one current value to another, Fig. 6. The function is specified

$$
\begin{aligned}
B\left(t_{n}, T_{w}\right)= & 0.42+0.5 \cos \left(\frac{\pi t_{n}}{T_{w}}\right) \\
& +0.08 \cos \left(\frac{2 \pi t_{n}}{T_{w}}\right)
\end{aligned}
$$

where $t_{n}$ is time normalized to the center of the window function and $T_{w}$ is the period of the function.

This is one of a group of finite-length window functions which are employed in digital signal processing [17]. Functions such as the Blackman window approximate the ideal window spectra with a finite-length.

The differential of the Blackman function is useful in assessing its suitability, Fig. 7. Clearly, the rate of change of the differential shown in Fig. 7 is non-zero for the standard function at $t_{n}=0$. This causes tracking errors for the current controller at the junction of the window and injection functions. A better match can be achieved with a modified window (Figs. 6, 7), albeit at a higher differential magnitude, and by shifting the tracking errors towards the $t_{n}=(-1,1)$ points. This is justified as the injection current at these points tends

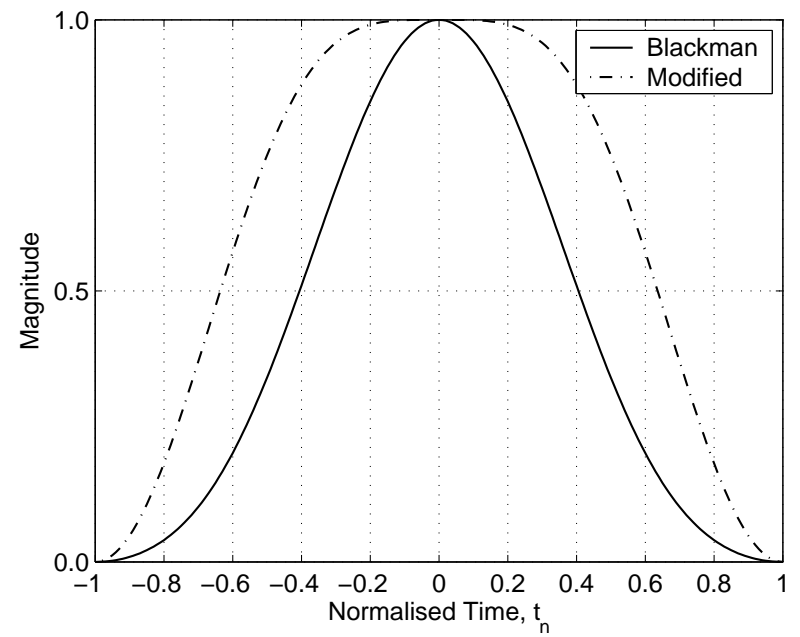

Fig. 6. Blackman-Type Windows

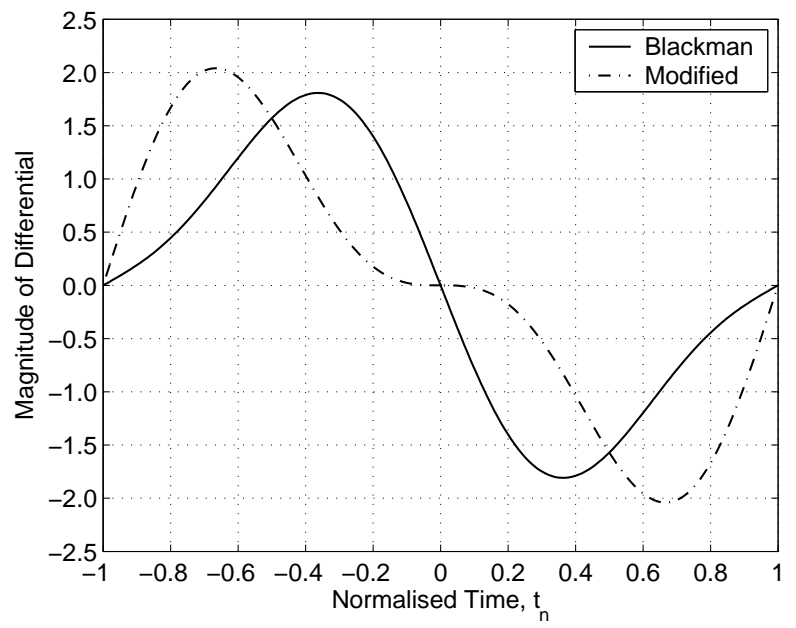

Fig. 7. Differential of Blackman-Type Windows

towards zero, and can play no part in resistance estimation. The modified function is given

$$
\begin{aligned}
B_{2}\left(t_{n}, T_{w}\right) & =0.625+0.5 \cos \left(\frac{\pi t_{n}}{T_{w}}\right) \\
& -0.125 \cos \left(\frac{2 \pi t_{n}}{T_{w}}\right) \\
\max \left(\frac{d(B)}{d t}\right) & =\frac{1.81}{T_{w}} \\
\max \left(\frac{d\left(B_{2}\right)}{d t}\right) & =\frac{2.04}{T_{w}} \\
\int_{-T_{w}}^{T_{w}} B(t) d t & =0.84 T_{w} \\
\int_{-T_{w}}^{T_{w}} B_{2}(t) d t & =1.25 T_{w}
\end{aligned}
$$

Using the maximum differential of the window profile, the machine-dependent drive voltage and current requirements per 


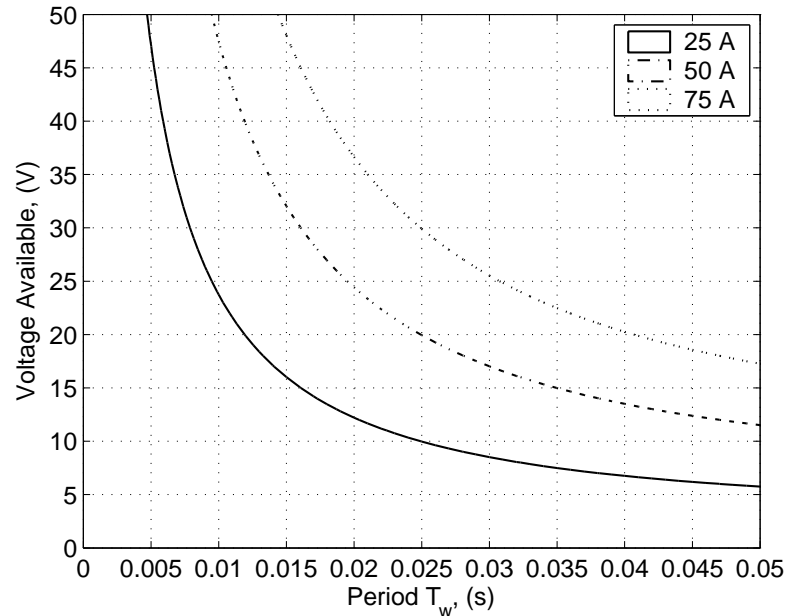

Fig. 8. Maximum Current Available for Injection with $L_{d}=9 \mathrm{mH}$ and $R_{s}$ $=0.133 \Omega$

phase can be calculated

$$
\begin{aligned}
V_{i n j} & =F \sqrt{\left(\frac{[1.81,2.04] L_{d}}{T_{w}}\right)^{2}+R_{s}^{2}} \\
I_{i n j} & =\frac{2}{3} F
\end{aligned}
$$

These are beneficial in specifying inverter VA rating for resistance estimation, or in tuning the window period $T_{w}$ to suit the available drive voltage. Given a certain maximum voltage available for injection and particular machine parameters, the maximum achievable injection current can be calculated for various $T_{w}$, Fig. 8 .

\section{InJeCtion CURREnt PRofile AND Power DISSIPATION}

Multiplying the window function in a piecewise manner with the injection currents (Eqs. $6 \& 18$ ) realize the injection current profiles, Fig. 10. The intermittent injection of the $d$ axis current through resistance $R_{s}$ causes resistive power loss

$$
\begin{gathered}
P_{F A}= \\
F^{2} \frac{N_{i n j} R_{s}}{T_{e l}}\left(\frac{2}{\omega} \sin \left(\frac{\omega T_{i}}{2}\right)+[0.84,1.25] T_{w}\right) \\
P_{F M}=\quad F^{2} \frac{N_{i n j} R_{s}}{T_{e l}}\left(T_{i}+[0.84,1.25] T_{w}\right)
\end{gathered}
$$

where $P_{F A}, P_{F M}$ are the average power losses for fixed-angle and fixed magnitude methods over elapsed time $T_{e l}, N_{i n j}$ is the number of injection current pulses in the elapsed time and $T_{i}$ is the duration of the current injection pulse. The values $[0.84,1.25]$ refer to the Blackman and modified windows respectively.

The practical significance of the losses introduced by current injection can be illustrated by reference to the PMSM of Table I which has a rated power of $11.9 \mathrm{~kW}$. By way of illustration, the additional losses generated as a function of current magnitude for the specific case of three injections every 10 seconds, with window $\operatorname{arc}$ of $\theta_{w}=10^{\circ}$ and injection arc of

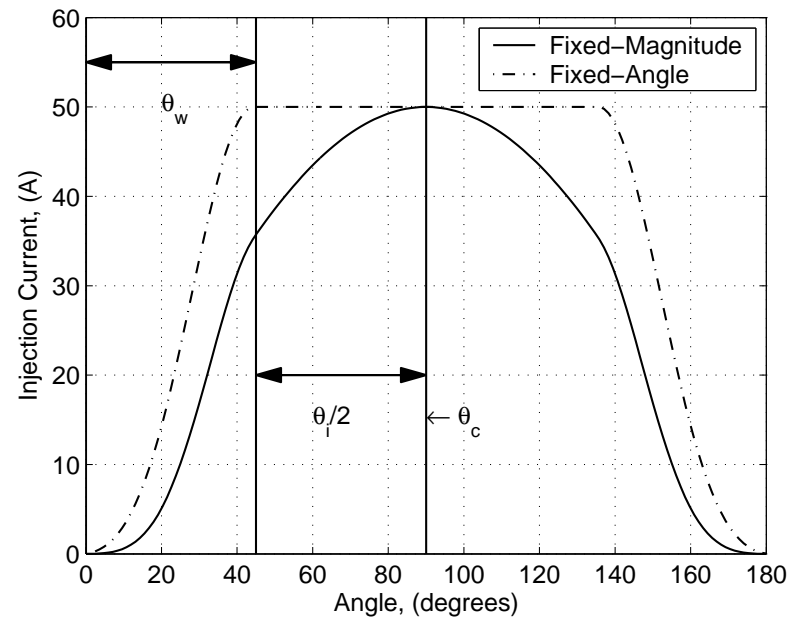

Fig. 9. Injection Current Profiles for $\theta_{i}=90^{\circ}$, where $\theta_{c}$ denotes the center angle for injection, and $\theta_{i}$ is the arc of the injection window

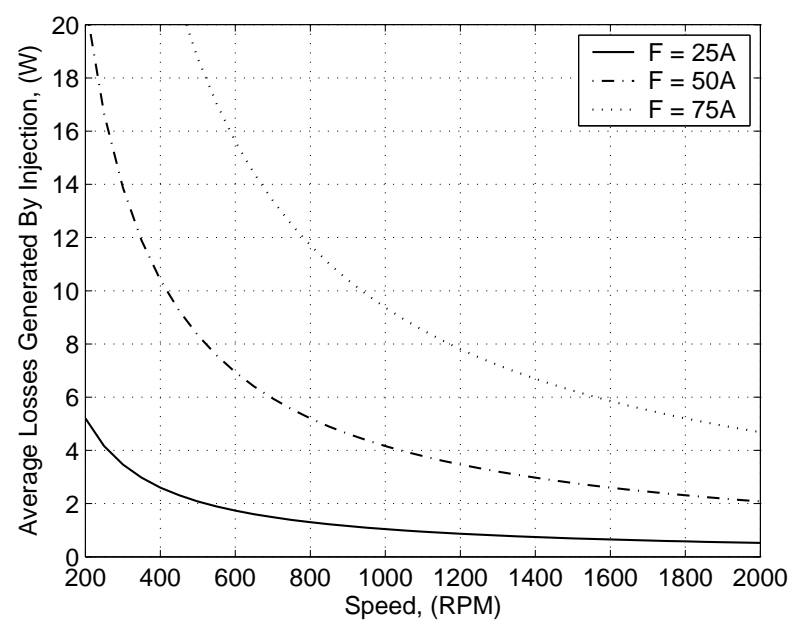

Fig. 10. Average Power Dissipation for Bipolar Injection

$\theta_{i}=720^{\circ}$ are plotted in Fig. 10. The intermittent estimation of resistance via current injection is sufficient for machines with long thermal time constants, and results in an additional heating load which is low when compared to the motor rated power.

\section{The Influence of The EMF}

The widespread use of the dq transform (Eq. 3) stems from its representation of the stator three-phase EMFs as a constant which is aligned with the $q$-axis in the rotor reference frame. The machine drive currents and EMFs must be sinusoidal for smooth, high bandwidth control.

Due to machine design constraints this ideal waveform cannot be achieved and the EMF may contain significant harmonics. Fig. 11 shows an ideal sinusoid and two EMF waveforms of the same peak voltage which are typical of those observed in practical machines [18], [19]. The 'Sinusoidal', 'Modular' and 'Trapezoidal' machines contain 100, 89 and 77 percent fundamental components respectively.

The EMF waveform has a significant effect on the axes voltages, especially at high speeds. The effect of non-sinusoidal 


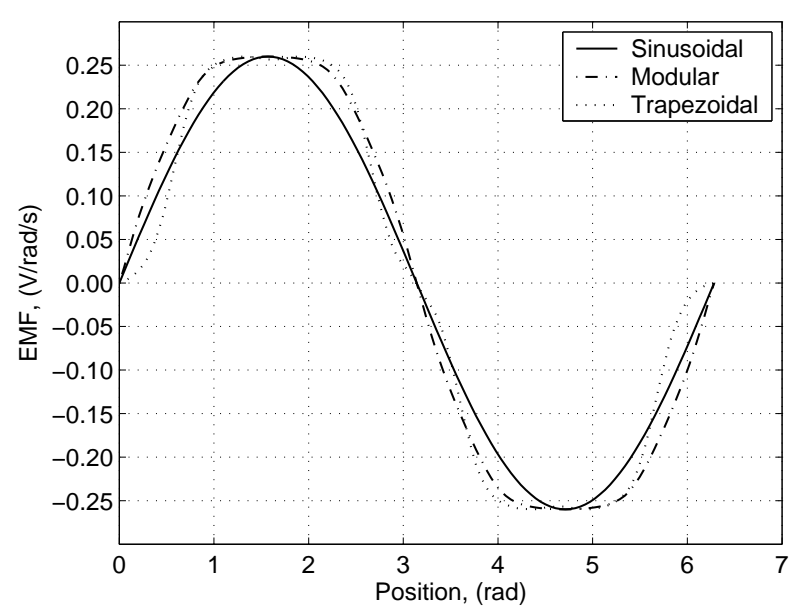

Fig. 11. Back-EMF Profiles

EMF on the axes voltages has been predicted by simulation of a vector-controlled drive-system similar to Fig. 3, and with the motor parameters of Table I. The fluctuation in axes voltages is comparable in magnitude to the resistive voltage under current injection. Harmonic generation from motors with lesssinusoidal EMF will be of greater magnitude, and resistance estimation will therefore be more difficult.

Methods employed to estimate the resistance must be able to separate the voltage harmonics from the superimposed signal voltage.

\section{Methods of Separating Injection Voltages FROM MOTORING VOLTAGES}

The injection voltages must be separated from the motoring (Eq. 3) and harmonic voltages. The unknown injection voltages can be defined if the motoring voltages and harmonics are defined.

The motoring voltages could be defined using parameters, requiring estimation procedures or empirical determination. The EMF harmonics in particular require many parameters to define. Basing the separation function on parameters is unwise, especially in a temperature-estimating drive system which may be subject to significant parameter deviation over the working temperature range.

The following methods are based on measuring the terminal voltage waveforms for a short interval during normal operation, i.e. with no current injection, and subtracting these waveforms from those obtained during a corresponding interval with current injection for the same nominal speed and motoring current conditions. Such approaches are inherently reliant on achieving near identical motoring conditions during the two intervals. Whilst this could be difficult to achieve in practical drive systems, there may be options to command the motor to a set torque or speed output during current injection.

During each interval the motor variables $i_{d r}, i_{q r}, v_{d r}, v_{q r}, \omega_{m}$ and $\omega$ are recorded and the difference in voltage is computed offline.

\section{A. Unipolar Method}

Only the second period contains an injection pulse, and the two periods are recorded over the same arc of rotor angle $\theta$. The harmonic voltages with respect to rotor position are assumed equal over two periods with unchanged motor parameters. Thus the difference in the recorded variables over the two time periods reveals the injection currents and voltages.

Estimation of harmonic voltages is thus avoided. From (Eq. 3), and neglecting the differential terms, the following equations can be formulated with arbitrary generated injection voltages $v_{i d r}$ on the d-axis, $v_{i q r}$ on the q-axis, and for two recordings of parameters starting at $\theta$ and $\theta+\Delta \theta$. For Recording 1:

$$
\begin{aligned}
v_{d r}(\theta)= & R_{s} i_{d r}(\theta)-\omega(\theta) L_{q} i_{q r}(\theta)+k_{d}(\theta) \omega_{m}(\theta) \\
v_{q r}(\theta)= & R_{s} i_{q r}(\theta)-\omega(\theta) L_{d} i_{d r}(\theta)+k_{q}(\theta) \omega_{m}(\theta) \\
& +K_{e} \omega_{m}(\theta)
\end{aligned}
$$

Recording 2:

$$
\begin{aligned}
v_{d r}(\theta+\Delta \theta)= & R_{s} i_{d r}(\theta+\Delta \theta) \\
& -\omega(\theta+\Delta \theta) L_{q} i_{q r}(\theta+\Delta \theta) \\
& +k_{d}(\theta+\Delta \theta) \omega_{m}(\theta+\Delta \theta)+v_{i d r}(\theta) \\
v_{q r}(\theta+\Delta \theta)= & R_{s} i_{q r}(\theta+\Delta \theta) \\
& -\omega(\theta+\Delta \theta) L_{d} i_{d r}(\theta+\Delta \theta) \\
& +k_{q}(\theta) \omega_{m}(\theta+\Delta \theta)+v_{i q r}(\theta+\Delta \theta)
\end{aligned}
$$

where $k_{d}(\theta), k_{q}(\theta)$ are the harmonic voltages with respect to electrical rotor position.

By subtraction of (Eq. 35 \& 36) from (Eq. $33 \&$ 34) the injection voltage is recovered providing that $k_{d}(\theta), k_{q}(\theta)=$ $k_{d}(\theta+\Delta \theta), k_{q}(\theta+\Delta \theta)$ (Eq. $37 \& 38$ ).

$$
\begin{aligned}
v_{i d r}(\theta)= & v_{d r}(\theta)-v_{d r}(\theta+\Delta \theta)+\Delta \omega L_{q} \Delta i_{q r} \\
& -\Delta i_{d r} R_{s} \\
v_{i q r}(\theta)= & v_{q r}(\theta)-v_{q r}(\theta+\Delta \theta)+\Delta \omega L_{d} \Delta i_{d r} \\
& -\Delta i_{q r} R_{s}+K_{e} \Delta \omega_{m}
\end{aligned}
$$

where $\Delta$ is the difference in the respective variables between measurement periods.

This equivalence can be achieved for electrical harmonics numbered 0 and upwards by setting $\Delta \theta=2 \pi$. The equivalence is also achievable for mechanical harmonics by setting $\Delta \theta=$ $2 p \pi$. The drawback is that $\Delta \theta$ is longer and the assumption that the motor parameters are unchanged may not be valid.

\section{B. Bipolar Method}

In the bipolar method, the first injection time period contains injection current of positive polarity, and the second of negative polarity. The EMF is separated as the recorded voltages and currents are subtracted from one another, and twice the magnitude of injection voltage and current is obtained. To obtain a specific signal magnitude the bipolar method therefore requires half the time period, or half the injection current magnitude of the unipolar. From Eq. 3, 
Recording 1:

$$
\begin{aligned}
v_{d r}(\theta)= & R_{s} i_{d r}(\theta)-\omega(\theta) L_{q} i_{q r}(\theta)+k_{d}(\theta) \omega_{m}(\theta) \\
& +v_{i d r}(\theta) \\
v_{q r}(\theta)= & R_{s} i_{q r}(\theta)-\omega(\theta) L_{d} i_{d r}(\theta)+k_{q}(\theta) \omega_{m}(\theta) \\
& +v_{i q r}(\theta)+K_{e} \omega_{m}(\theta)
\end{aligned}
$$

Recording 2:

$$
\begin{aligned}
v_{d r}(\theta+\Delta \theta)= & R_{s} i_{d r}(\theta+\Delta \theta) \\
& -\omega(\theta+\Delta \theta) L_{q} i_{q r}(\theta+\Delta \theta) \\
& +k_{d}(\theta+\Delta \theta) \omega_{m}(\theta+\Delta \theta)-v_{i d r}(\theta) \\
v_{q r}(\theta+\Delta \theta)= & R_{s} i_{q r}(\theta+\Delta \theta) \\
& -\omega(\theta+\Delta \theta) L_{d} i_{d r}(\theta+\Delta \theta) \\
& +k_{q}(\theta) \omega_{m}(\theta+\Delta \theta)-v_{i q r}(\theta+\Delta \theta)
\end{aligned}
$$

By subtraction twice the injection voltage is recovered

$$
\begin{aligned}
2 v_{i d r}(\theta)= & v_{d r}(\theta)-v_{d r}(\theta+\Delta \theta)+\Delta \omega L_{q} \Delta i_{q r} \\
& -\Delta i_{d r} R_{s} \\
2 v_{i q r}(\theta)= & v_{q r}(\theta)-v_{q r}(\theta+\Delta \theta)+\Delta \omega L_{d} \Delta i_{d r} \\
& -\Delta i_{q r} R_{s}+K_{e} \Delta \omega_{m}
\end{aligned}
$$

Both methods rely on the repeatability of measurements made with respect to rotor position from one injection period to the next. If there is sufficient change in any of the system conditions then the methods are likely to become erroneous. Monitoring the speed change $\Delta \omega$ between the injection periods could provide a means of predicting the suitability of the data for resistance estimation.

From the determinations of the injection voltages $v_{i d r}$ and $v_{i q r}$, and knowledge or measurement of the injected currents, the stator winding resistance can be estimated from (Eqs. 20 \& 14).

\section{FiXed-Angle versus FiXed-Magnitude}

For the fixed-angle method, the resistance may be estimated from the determined resistive signal voltages (Eq. 22) using the phase and magnitude of detected injection voltage. Due to the changing magnitude of resistive signal voltage, only a small number of samples per revolution can be obtained relative to the number of measurements that can be made in a whole revolution of the rotor.

This makes it likely that many revolutions will be required in order to achieve adequate separation of the resistive signal from noise and error voltages. There may also be a reduction in injection current magnitude due to attenuation of the sinusoidal current by the current controller.

For the fixed-magnitude method the resistive voltage (Eq. 20) is constant. Thus the injection current may be applied for an indefinite, multi-revolution period of time. As the injection current is constant in the current-controller frame, there will be no problem with attenuation. There will be deviations in the detected resistive voltage due to noise and variation of the system conditions between the two time intervals, and the application of filters to smooth these out is straightforward with the underlying resistive voltage being
TABLE I

PMSM PARAMETERS

\begin{tabular}{|l|l|l|}
\hline Parameter & Symbol & Value \\
\hline Torque constant & $K_{t}$ & $2.08 \mathrm{Nm} / \mathrm{A}$ \\
\hline EMF constant & $K_{e}$ & $1.19 \mathrm{Vs} \mathrm{radian}^{-1}$ \\
\hline Rated speed & $\omega_{\text {rated }}$ & $314 \mathrm{radians}^{-1}$ \\
\hline Rated continuous power output & $P_{\text {cont }}$ & $11.9 \mathrm{~kW}$ \\
\hline Rated continuous current & $I_{\text {cont }}$ & $31.9 \mathrm{~A} \mathrm{RMS}$ \\
\hline Stator resistance & $R_{s}$ & $0.133 \Omega$ \\
\hline Stall current & $I_{s t a l l}$ & $117.5 \mathrm{~A} \mathrm{RMS}$ \\
\hline Maximum phase voltage & $V_{a c}$ & $480 \mathrm{~V}$ \\
\hline Line-to-line inductance & $L_{l-l}$ & $11 \mathrm{mH}$ \\
\hline Rotor moment of inertia & $J$ & $0.00841 \mathrm{~kg} \mathrm{~m}^{2}$ \\
\hline Pole-pairs & $p$ & 3 \\
\hline Thermal time constant & $\tau_{t h}$ & $2880 \mathrm{~s}(48 \mathrm{~min})$ \\
\hline Torque due to Coulomb friction & $T_{c}$ & $0.908 \mathrm{Nm}$ \\
\hline
\end{tabular}

constant. Comparing the d-axis current injection methods with the DC injection method, the d-axis methods do not produce the torque ripple that is a consequence of the DC offset. The unipolar and bipolar methods provide a means to separate the injection current and voltage signals which is not dependent on frequency separation, but on the likelihood that change in the motoring system conditions between two time intervals is small.

\section{CONCLUSIONS}

Two methods of current injection have been presented which do not directly generate torque in a PMSM. These methods are suitable for stator resistance estimation in machines with relatively low resistances compared to terminal voltage magnitude, by virtue of the decoupling of the resistance-sensing current from the motor's torque-producing current. A novel separation technique is presented which is able to recover the voltage generated by current injection from two recordings of motor parameters; without the estimation of many of the PMSM parameters being required, and without the need for frequency separation between motoring and injection currents. A window function is also presented which smooths transitions between the high current magnitudes required for resistance-sensing and the lower magnitudes of motoring currents. The authors will publish a companion paper, detailing the application of the bipolar current injection technique to a PMSM. The technique was able to maintain a temperature estimation error of less than $10^{\circ} \mathrm{C}$ at winding temperatures above $60^{\circ} \mathrm{C}$.

\section{REFERENCES}

[1] A. H. Bonnet, "Root cause ac motor failure analysis," IEEE Trans. Ind. Appl., vol. 36, no. 5, pp. 1435-1448, Sep./Oct. 2000.

[2] R. Gleichman, "Failure modes and field testing of medium-voltage motor windings," IEEE Trans. Ind. Appl., vol. 38, no. 5, pp. 1473-1476, Sep./Oct. 2002.

[3] S. Farag, R. Bartheld, and T. Habetler, "An integrated on-line motor protection system," IEEE Trans. Ind. Appl., vol. 2(2), pp. 21-6, Mar./Apr. 1996.

[4] R. Kerkman, B. Seibel, T. Rowan, and D. Schlegel, "A new flux and stator resistance identifier for ac drive systems," IEEE Trans. Ind. Appl., vol. 32, no. 3, pp. 585-593, May/Jun. 1996.

[5] M. Elbuluk, L. Tong, and I. Hussain, "Neural-network-based model reference adaptive systems for high performance motor drives and motion controls," IEEE Trans. Ind. Appl., vol. 38, no. 3, pp. 879-886, Jun. 2002. 
TABLE II

SYMBOL TABLE

\begin{tabular}{|c|c|}
\hline Symbol & Parameter \\
\hline$\alpha$ & Temperature coefficient of copper \\
\hline$\beta$ & Regulation factor \\
\hline$\Delta$ & Change in a parameter \\
\hline$\theta$ & Electrical rotor position \\
\hline$\theta_{F}$ & Angle of injected current vector (elec) \\
\hline$\theta_{L}$ & Angle of resistive voltage in stator frame (elec) \\
\hline$\theta_{m}$ & Mechanical rotor position (mech) \\
\hline$\theta_{R}$ & Angle of inductive voltage in stator frame (elec) \\
\hline$\theta_{q d s}$ & Angle of resistive and inductive voltage in stator frame (elec) \\
\hline$\omega$ & Electrical frequency \\
\hline$\omega_{m}$ & Mechanical (shaft) frequency \\
\hline$B$ & Blackman window function \\
\hline$B_{2}$ & Modified Blackman window function \\
\hline$F$ & Magnitude of injected current vector \\
\hline$\overline{i_{a}}$ & Phase-A current \\
\hline$i_{b}$ & Phase-B current \\
\hline$i_{c}$ & Phase-C current \\
\hline$i_{d r}$ & D-axis current, rotor reference frame \\
\hline$\overline{i_{q r}}$ & Q-axis current, rotor reference frame \\
\hline$i_{d s}$ & D-axis current, stator reference frame \\
\hline$i_{q s}$ & Q-axis current, stator reference frame \\
\hline$k_{d}$ & Coefficient for D-axis EMF harmonics \\
\hline$k_{q}$ & Coefficient for D-axis EMF harmonics \\
\hline$K_{e}$ & EMF constant \\
\hline$\overline{K_{t}}$ & Torque constant \\
\hline$L_{d}$ & D-axis inductance \\
\hline$L_{q}$ & Q-axis inductance \\
\hline$N_{i n j}$ & Number of injected current pulses \\
\hline$P_{F A}$ & Power dissipation for fixed-angle method \\
\hline$P_{F M}$ & Power dissipation for fixed-magnitude method \\
\hline$R_{s}$ & Stator resistance \\
\hline$R_{s 0}$ & Stator winding resistance at reference temperature \\
\hline$S_{0}$ & Zero-sequence quantity \\
\hline$S_{a}$ & Phase-A quantity \\
\hline$S_{b}$ & Phase-B quantity \\
\hline$S_{c}$ & Phase-C quantity \\
\hline$S_{d}$ & D-axis quantity, rotor reference frame \\
\hline$S_{q}$ & Q-axis quantity, rotor reference frame \\
\hline$T_{0}$ & Reference temperature \\
\hline$T_{e l}$ & Elapsed time between injected pulses \\
\hline$T_{e m}$ & Electromagnetic torque \\
\hline$T_{i}$ & Duration of current injection pulse \\
\hline$T_{s}$ & Stator winding temperature \\
\hline$T_{w}$ & Time-period of the window function \\
\hline$v_{i d r}$ & D-Axis voltage caused by current injection \\
\hline$v_{\text {iqr }}$ & Q-axis voltage caused by current injection \\
\hline$v_{d r}$ & D-axis voltage, rotor reference frame \\
\hline$v_{q r}$ & Q-axis voltage, rotor reference frame \\
\hline$V_{L}$ & Magnitude of inductive voltage \\
\hline$V_{R}$ & Magnitude of resistive voltage \\
\hline$V_{q d s}$ & Magnitude of resistive and inductive voltage in stator frame \\
\hline
\end{tabular}

[6] J. Campbell and M. Sumner, "An artificial neural network for stator resistance estimation in a sensorless vector controlled induction motor drive," 9th European Conference on Power Electronics and Applications, vol. 2001, Graz, Austria Aug.

[7] L. Umanand and S. Bhat, "Online estimation of stator resistance of an induction motor for speed control applications," IEE Proc.-Electr. Power Appl., vol. 142, no. 2, pp. 97-103, Mar. 1995.

[8] V. Vasic, S. Vukosavic, and E. Levi, "A stator resistance estimation scheme for speed sensorless rotor flux oriented induction motor drive," IEEE Trans. Energy Convers., vol. 18, no. 4, pp. 476-482, Dec. 2003.

[9] B. Bose and N. Patel, "Quasi-fuzzy estimation of stator resistance of induction motor," IEEE Trans. Power Electron., vol. 18, no. 4, pp. 401409, May 1998.

[10] L. C. Benn, B. Burton, and R. G. Harley, "Online stator resistance estimation for thermal monitoring of converter fed induction motors using pseudorandom modified pwm," in Conference Record of the 2004 IEEE Industry Applications Conference. 39th IAS Annual Meeting, Oct.
2004, pp. 727-34

[11] B. Nahid-Mobarakeh, F. Meibody-Tabar, and F.-M. Sargos, "Mechanical sensorless control of pmsm with online estimation of stator resistance," IEEE Trans. Ind. Appl., vol. 40(2), pp. 457-71, Mar./Apr. 2004.

[12] P. Milanfar and J. H. Lang, "Monitoring the thermal condition of permanent-magnet synchronous motors," IEEE Trans. Aerosp. Electron. Syst., vol. 32(4), pp. 1421-9, Oct. 1996.

[13] S. Lee and T. Habetler, "An online stator winding resistance estimation technique for temperature monitoring of line connected induction machines," IEEE Trans. Ind. Appl., vol. 39, no. 3, pp. 685-694, May/Jun. 2003.

[14] C. Jacobina, J. Filha, and A. Lima, "On-line estimation of the stator resistance of induction machines based on zero sequence model," IEEE Trans. Power Electron., vol. 15, no. 2, pp. 346-352, Mar. 2000.

[15] A. Fitzgerald, J. Charles Kingsley, and S. D. Umans, Electric Machinery. McGraw-Hill, 2003.

[16] P. Stewart and V. Kadirkamanathan, "Dynamic model tracking design for low inertia, high speed permanent magnet ac motors," ISA Transactions, vol. 43, no. 1, pp. 111-22, Jan. 2004

[17] S. D. Stearns and D. R. Hush, Digital Signal Analysis, 2nd ed. PrenticeHall, 1990, p. 157.

[18] J. Wang, Z. .Xia, and D. Howe, "Analysis of three-phase surfacemounted magnet modular permanent magnet machines," IEEE Trans. Power Electron., vol. 3, pp. 27-32, 2004.

[19] Z. Zhu, D. Howe, and B. Ackermann, "Analytical prediction of dynamic performance characteristics of brushless dc drives," Electric Machines and Power Systems, vol. 20, no. 6, pp. 661-678, 1992.

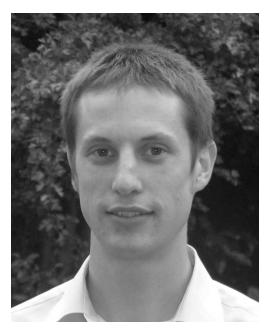

Simon Delamere Wilson was born in Chester, Cheshire, England in 1979. He received the M.Eng degree in Electrical Engineering from the University of Sheffield, UK, in 2004 and his $\mathrm{PhD}$ from the same institution in 2009. Since 2007, he has been a Consultant in the New Energy Technologies department at EA Technology, Capenhurst, Chester. His research interests include power electronics, drive systems and renewable energy and energy efficiency.

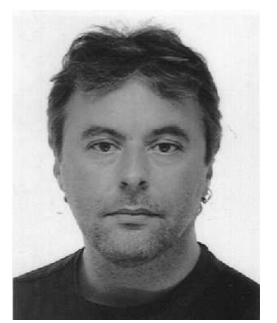

Paul Stewart Paul Stewart (M'2000-SM'2004) was born in Birmingham, England in 1960. He received the B.Eng and Ph.D degrees in electronic, control and systems engineering from the University of Sheffield UK, in 1996 and 2000 respectively. He became Assistant Professor in Electronic and Electrical Engineering in the Machines and Drives Group at the University of Sheffield in 2002, and Associate Professor in 2007. He is currently Founding Head of Engineering at the University of Lincoln. His research interests are in multiobjective optimisation and intelligent control with automotive and aeronautic applications.

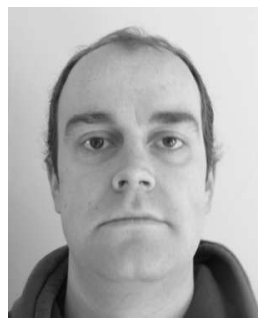

Ben Taylor received the M.Eng degree in electronic engineering (communications) from the University of Sheffield, Sheffield, UK, in 1998. Currently, he is employed as a research associate in the Department of Automatic Control and Systems Engineering, at the University of Sheffield, working on power systems modelling for more electric aircraft platforms. His research interests also include power electronics, machine control and energy scavenging systems. 